\title{
Review on a palm vein infrared image acquisition system
}

\begin{abstract}
Palm vein recognition system is one of the modalities in biometric technology. It manipulates the vast pattern of vein under the palm as one of the measurements in validating a person with his or her claimed identity. One of the components in a palm vein recognition system is the image acquisition part. With a suitable configuration, the image acquisition system may capture the required image, just enough for the recognition process. This paper is a brief review on the development needs of a palm vein image acquisition system.
\end{abstract}

Keyword: Image acquisition; Image analysis; Vein biometrics 